\title{
Automated Segmentation of Dental Calculus in Optical Coherence Tomography Images
}

\author{
Chia-Yen Lee, ${ }^{1 *}$ Ching-Cheng Chuang, ${ }^{2}$ Guan-Jie Chen, ${ }^{1}$ \\ Chih-Chia Huang, ${ }^{3}$ Shyh-Yuan Lee, ${ }^{4}$ and Yu-Hsien Lin ${ }^{5}$
}

\footnotetext{
${ }^{1}$ Department of Electrical Engineering, National United University, Miaoli, Taiwan, R.O.C. ${ }^{2}$ Institute of Biomedical Engineering, National Chiao Tung University, Hsinchu, Taiwan, R.O.C. ${ }^{3}$ Endodontology Department of Dentistry, Cardinal Tien Hospital, New Taipei, Taiwan, R.O.C. ${ }^{4}$ Department of Dentistry, National Yang Ming University, Taipei, Taiwan, R.O.C.

${ }^{5}$ Department of Electronic Engineering, National United University, Miaoli, Taiwan, R.O.C.
}

(Received March 28, 2018; accepted August 31, 2018)

Keywords: optical coherence tomography (OCT), image analysis, segmentation, dental calculus

The presence of dental calculus is highly correlated with the formation and advancement of periodontal disease. The occurrence and relapse of periodontal disease can be prevented only if dental calculus is completely removed. In this study, optical coherence tomography (OCT) is used to obtain two-dimensional cross-sectional images of tooth samples, in conjunction with a segmentation technique that enables automatic identification of dental calculus. We propose the vertical intensity transform function to correct the nonuniform instrument signal intensity caused by OCT. Afterwards, the detection ranges are defined by K-means or the Markov random field (MRF), and the candidate range is selected on the basis of mathematical morphology (MM). Finally, the features (thickness gradient, texture, and tooth surface slope) are quantified, and dental calculus is recognized and segmented. In the preliminary result, the sensitivity is $87.5 \%$. The mean distance between the boundaries generated by the proposed algorithm and the corresponding manually delineated boundaries is $2.52 \pm 3.54$ pixels. Our proposed algorithm assists physicians to determine dental calculus more easily. Doctors no longer need to rely solely on their experiences to recognize dental calculus, but can refer to specific data to assist in diagnosis.

\section{Introduction}

According to the investigation by the Taiwan Association of Medical Screening, the incidence rate of periodontal disease among adults over 18 years of age is as high as $99.29 \%$, and that of dental calculus is $40.66 \% .^{(1)}$ Dental calculus is highly correlated with the formation and advancement of periodontal disease. ${ }^{(2)}$ Dental plaque is the primary cause of periodontal disease. Degenerated dental plaque and mineral substances in the saliva are mixed and hardened to form dental calculus. The rough surface provides bacteria with a suitable breeding environment, thus continuously harming the periodontal tissue. The occurrence and relapse of

*Corresponding author: e-mail: leecyya@gmail.com

https://doi.org/10.18494/SAM.2018.2053 
periodontal disease can be prevented only if dental calculus is completely removed. However, dental calculus is not easily observed. Therefore, a detection technique that can enable accurate detection and quantification of dental calculus is the key to preventing and treating periodontal diseases.

Optical coherence tomography (OCT) is a noninvasive, nonradiation, and highresolution optical technique that can capture cross-sectional images of biological tissues instantly. The application of OCT in medical fields has been studied for years, for example, gastroenterology, ${ }^{(3,4)}$ ophthalmology, ${ }^{(5-7)}$ dermatology, ${ }^{(8,9)}$ and dentistry ${ }^{(10,11)}$ where there have been comments on cariosity, periodontal disease, oral cancer foci, ${ }^{(10)}$ and the measurement of the refractive index of enamel, dentin, and cementum and dental calculus. ${ }^{(11)}$ The applications are in judging and quantifying the characteristics of tooth tissue and foci in the OCT image. Hundreds of two-dimensional cross-sectional images of a tooth obtained by OCT can be restructured into a three-dimensional image. However, it is still difficult and time-consuming for doctors to recognize the location of dental calculus by observing the tooth surface and brightness variation in the hundreds of two-dimensional cross-sectional images, such as Fig. 1. In this study, we developed an algorithm to automatically identify and quantify dental calculus in two-dimensional OCT images and then reconstruct a three-dimensional image to assist doctors in clinical diagnosis.

The processing of medical images consists of several standard procedures, namely, image preprocessing, image segmentation, and extraction and segmentation of image features. Image preprocessing, i.e., spatial filtering, ${ }^{(12,13)}$ histogram equalization, ${ }^{(14,15)}$ and intensity transformation, ${ }^{(16)}$ changes the intensity and contrast of the entire image. However, the intensity derived from OCT signals depends on the optical attenuation. Therefore, the above-mentioned image preprocessing methods cannot be directly applied to the OCT images. Aiming at the attenuation characteristics in OCT images, we propose a normalization method, called the vertical intensity transform function (VITF), to adjust the intensity of the OCT image in the vertical direction to the same intensity level. This method can solve the problem of signal attenuation that causes vertical inhomogeneity.

The detection of dental calculus belongs to the process of image segmentation. The common image segmentation methods include thresholding, ${ }^{(17)}$ watershed, ${ }^{(18,19)}$ level set, ${ }^{(20)}$ region growing, ${ }^{(21)}$ and active contour modeling. ${ }^{(22)}$ Those methods require distinct boundary and gray levels of the target or a significant difference between the gray level gradient and the

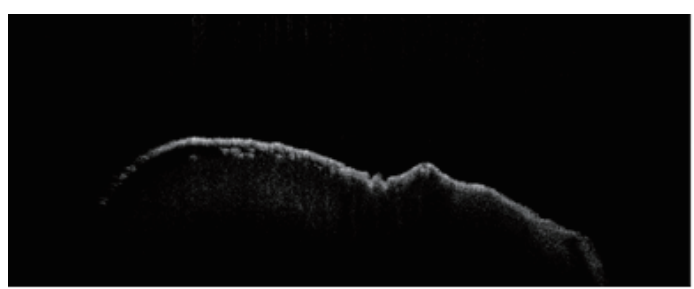

(a)

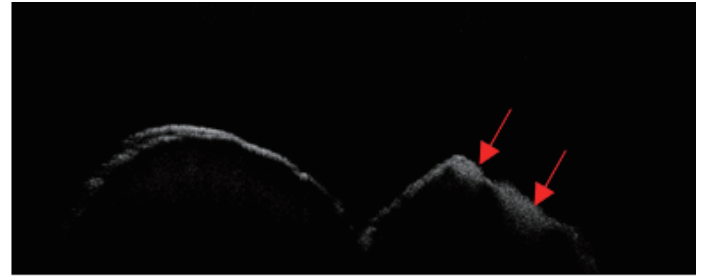

(b)

Fig. 1. (Color online) OCT images (a) without dental calculus and (b) with dental calculus shown by red arrows. 
background. However, in the OCT image, the boundary between dental calculus and other tooth tissues is fuzzy, and there is speckle noise. Therefore, general image segmentation methods are inapplicable to tooth OCT images.

In this study, K-means, Markov random field (MRF), and mathematical morphology (MM) were used to first narrow the detection ranges in accordance with the characteristics of different dental tissues and then to perform texture analysis on the detection ranges to segment dental calculus.

\subsection{Dental calculus detection ranges}

The K-means algorithm is a clustering algorithm that yields the optimum cluster by iterating the optimization approach. ${ }^{(23)}$ Patel and Sinha proposed the adaptive K-means algorithm to determine the focal area of breast cancer in a breast X-ray image. ${ }^{(24)}$ Sruthi used the K-means algorithm to identify the tumor region in magnetic resonance images of the brain on the basis of the average gray level. ${ }^{(25)}$ Since dental calculus only occurs in the tooth surface layer, where the tooth tissue has a high gray level, the K-means algorithm is applicable to segmenting the tooth surface layer. However, the detection ranges usually have similar properties. The $\mathrm{MRF}^{(26)}$ is a probabilistic model that can predict the category of the regions.

\subsection{Segmentation of dental calculus}

In order to further segment dental calculus in these dental calculus detection ranges, we adopt $\mathrm{MM}^{(27)}$ in which the too thin layer, i.e., not dental calculus, is removed first. For the rest of the detection ranges, the segmentation method is designed in accordance with the four different tooth tissue features: (1) the dentin and enamel blocks have different aspect ratios (ARs); (2) dental calculus on the dentin block thickens the tooth; (3) enamel and dental calculus have different gray scale distributions after binarization; and (4) dental calculus causes the protrusion of the tooth surfaces. The feature values of $A R \mathrm{~s}$, thickness, and tooth surface slope can be obtained by quantifying the four features. In addition, we also used texture analysis, ${ }^{(28)}$ which is often used in a quantified space structure, such as entropy, ${ }^{(29,30)}$ for identifying dental calculus.

\section{Materials and Methods}

The core of the algorithm proposed in this study is the definition of dental calculus regions on the basis of the respective characteristics of dentin, enamel, and calculus, and to further identify dental calculus in these areas. Figure 2 shows the flowchart of the segmentation algorithm. First, the VITF algorithm was used to improve the problem of the gray scale differences on the tooth surfaces owing to optical attenuation. Because dental calculus only appears in the surface area of teeth with a high gray scale, using the K-means algorithm with the MRF can reveal the ranges with higher gray scales, i.e., the tooth surfaces, that are required to be characterized for segmentation in the next step. 

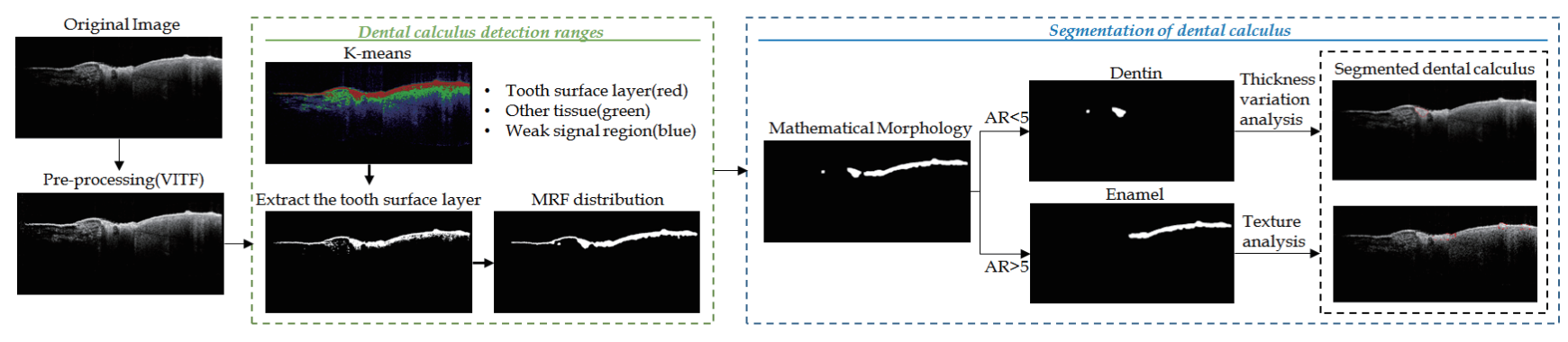

Fig. 2. (Color online) Flowchart of the proposed segmentation algorithm.

After the range is defined, the part of the tooth surface layer that is too thin to have dental calculus can be removed directly. The candidate regions of dental calculus are obtained by MM to remove the excessively thin surface layer and then are divided into dentin and enamel blocks on the basis of the image features of dental calculus accumulated on different tooth tissues. As the signal attenuates rapidly when passing through the dentin, this part is long and wide and has a low $A R$. The dentin is apparently thickened because there is dental calculus on it. The enamel is thick so the thickness variation caused by dental calculus is not obvious. The region with dental calculus causes the protrusion of the tooth surface layer, and the surface slope is changed. For the different features of dental calculus in the two regions, the segmentation methods should be designed accordingly. The aforesaid features can be summarized into the following three points: (1) the dentin and enamel blocks can be distinguished from the $A R$ of the blocks remaining after removing the excessively thin surface layer; (2) the surface layer thickness (vertical height) variation is used for judging dental calculus in the dentin block; and (3) dental calculus in the enamel block is judged on the basis of the tooth surface slope changes.

The dentin block has a low $A R$. In this type of block, the surface layer is thickened, probably owing to dental calculus or noise, so that it is not removed by MM. However, a comparison of the thickness variations of the block and the peripheral surface layer enables dental calculus, which has large variations, to be distinguished from the general surface layer, which has small variations. The enamel block has a high $A R$. It is not easy to distinguish between enamel and dental calculus by thickness variation analysis. Therefore, the texture feature (entropy) and the change in the tooth surface slope after binarization are applied to the enamel block for segmenting dental calculus.

\subsection{Image preprocessing}

The signal attenuates as the distance between the tooth and instrument increases, so that the height difference of the tooth surface becomes representative of the gray level difference. Therefore, the gray level increases towards the upper side of the OCT image, as shown in Fig. 3(a). In order to normalize the gray level of the tooth and retain the original contrast ratio, the VITF is proposed in accordance with the principle of the intensity transformation function, expressed as 


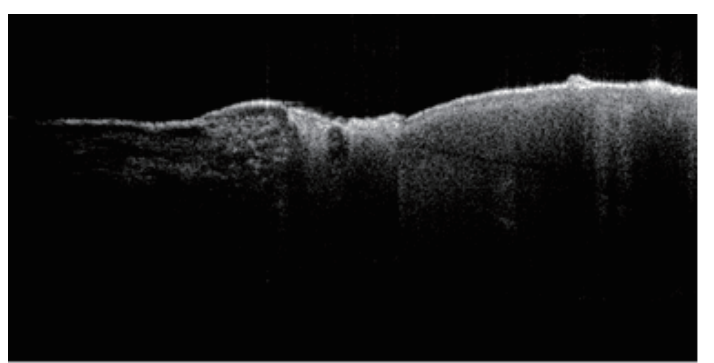

(a)

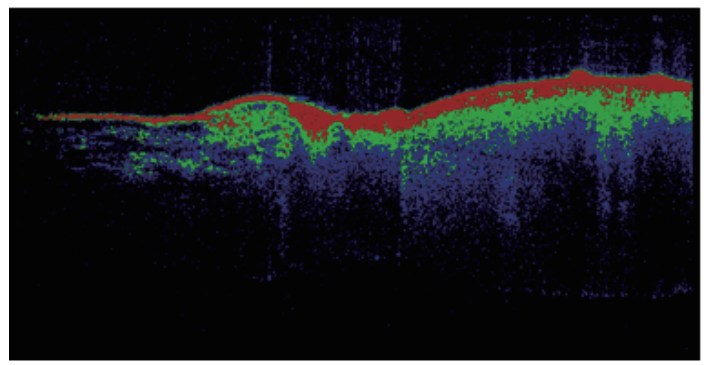

(c)

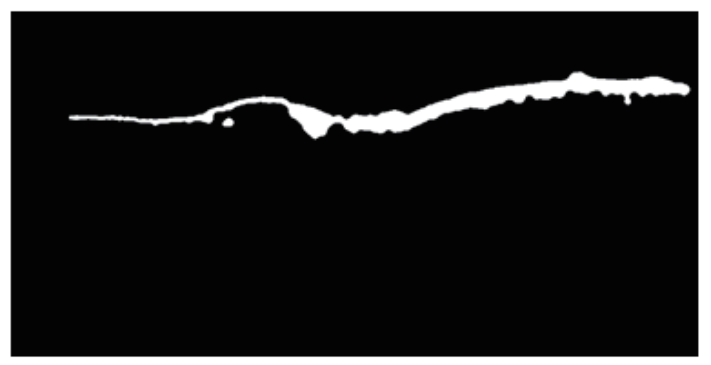

(e)

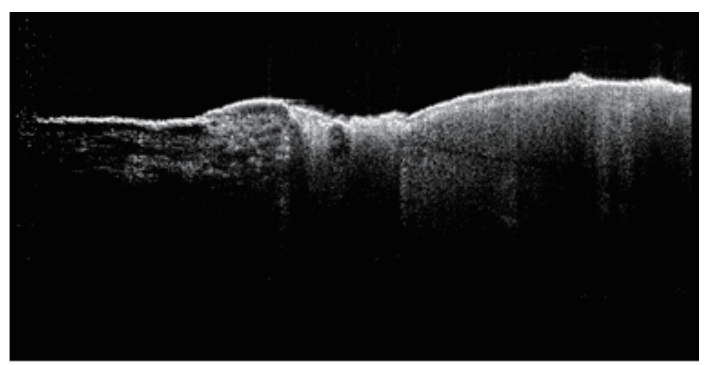

(b)

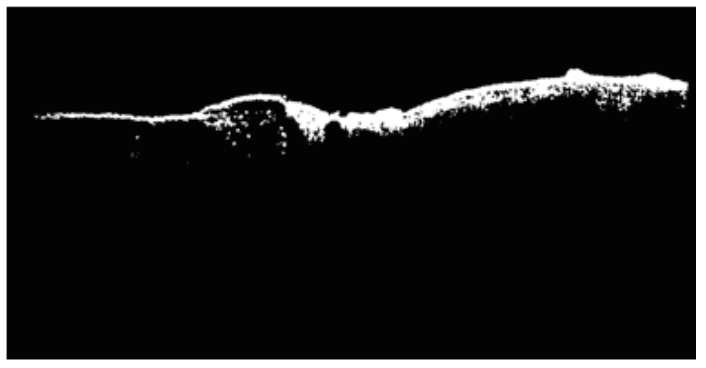

(d)

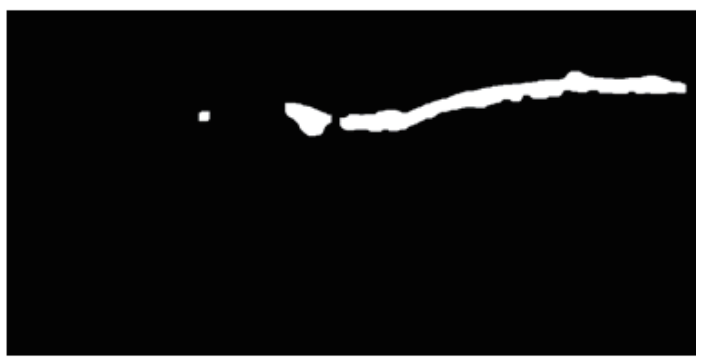

(f)

Fig. 3. (Color online) Results of each step in the process of defining dental calculus ranges. (a) Original image $I$. (b) Normalized image NI. (c) The clusters of K-means: tooth surface layer (red), other tooth tissues (green), and weak signal region (blue). (d) Results of cluster analysis of K-means algorithm. (e) MRF probability distribution MRFI. (f) Candidate block of dental calculus $O I$ determined by MM.

$$
N I(i, j)=\frac{I(i, j)}{\max _{k}(I(k, j))} \times 255,
$$

where the maximum value of each row of pixels of the original image $I$ is adjusted to 255 and the other pixels of the same row are adjusted proportionally, whereby the normalized image $N I$ is obtained, as shown in Fig. 3(b). $i=1,2,3, \ldots, n$ columns, $j=1,2,3, \ldots, n$ rows, and $k$ is the column number of the maximum value of pixels in row $j$. 


\subsection{Dental calculus detection ranges}

Dental calculus only accumulates on the tooth surface, so the preliminarily selected candidate region of dental calculus in the OCT image is concentrated only on the tooth surface layer. The instrument signal received from the tooth surface layer is the strongest, meaning the region has the maximum average gray level. The K-means algorithm is a clustering algorithm that determines the optimum center of gravity and produces clusters on the basis of the initial center of gravity, expressed as

$$
\underset{S}{\arg \min } \sum_{a=1}^{b} \sum_{x \in S_{a}}\left\|x-u_{a}\right\|^{2}
$$

where $x=\left(x_{1}, x_{2}, x_{3}, \ldots, x_{T}\right)$ is the average gray level of the region of pixels with nonzero gray levels, $u_{a}$ is the center of gravity of all elements in a cluster. The three convergent regions, the brightest tooth surface layer, other tooth tissues, and the weak signal region, can be found by iteration of Eq. (2), as shown in Fig. 3(c). However, since dental calculus only accumulates on the tooth surface, only the tooth surface layer is selected as the candidate region of dental calculus, as shown in Fig. 3(d).

According to the result of the K-means algorithm [Fig. 3(d)], the region extracted by the K-means algorithm matches the objective, but the region is too scattered owing to speckle noise and thus, is not a complete region. In order to further converge the candidate region of dental calculus, the MRF can be used to correct the tooth surface layer probability distribution. The basic concept of the MRF algorithm is to determine its own state from the probability of occurrence of different states in the random field, expressed as

$$
\begin{aligned}
& p=\frac{1}{c} \sum f_{r}\left(y_{\{r\}}\right), \\
& y_{\{r, v\}}= \begin{cases}p>0.5, & 1, \\
p<0.5, & 0,\end{cases}
\end{aligned}
$$

where each pixel $y_{\{r, v\}}$ has a random field $f_{r}$ and $y_{\{r\}}=y_{\{r, 1\}}, y_{\{r, 2\}}, y_{\{r, 3\}}, \ldots, y_{\{r, c\}}$ is the state of all random variables in the random field. There are only two states: tooth surface layer and not tooth surface layer. The state of maximum probability is the state of this pixel. The optimum probability distribution can be obtained by iterating Eqs. (3) and (4), as shown in Fig. 3(e). In the selected candidate region of dental calculus, most of the noise interference is removed, and the upper and lower boundaries of dental calculus are determined.

\subsection{Segmentation of dental calculus}

As different feature values are required for segmenting dental calculus on different tooth tissues, to distinguish different tooth tissues, we use the MM where only the specified structure 
is removed, as expressed in Eq. (5). The excessively thin region of the tooth surface layer where dental calculus cannot exist is removed. For the remaining candidate blocks of dental calculus, the segmentation method is selected in accordance with the $A R$. The $A R$ is calculated by dividing the square of distance $w_{d}$ between the line number left $t_{d}$ of the leftmost pixel and the line number right $t_{d}$ of the rightmost pixel in the $d$ th block by the area $A_{d}$, as expressed in Eq. (6). The threshold of $A R$ approximately distinguishes between dentin and enamel. The purpose is to prevent noise from thickening the tooth surface layer. The dentin not removed by MM is assigned to texture analysis, causing mis-segmentation. The blocks identified by the threshold are shown in Fig. 3(f).

$$
\begin{aligned}
& O I=(M R F I ! O) \oplus O \\
& A R=\frac{w_{d}{ }^{2}}{A_{d}}
\end{aligned}
$$

\section{Feature extraction and screening}

When the $A R$ of the dentin block is smaller than the threshold, method (1) is used. When the $A R$ of the enamel block is greater than the threshold, method (2) is used.

Method (1): Thickness Variation Analysis. Dental calculus on the dentin block with $A R$ smaller than the threshold [Fig. 4(a)] is obvious. The difference $\nabla L$ between the average vertical height $\left(A_{d} / w_{d}\right)$ of the block and the average vertical height $\left(h_{d}\right)$ of the peripheral dentin surface layer is obvious. $l$ is the length of the range of a general tooth surface layer used as a reference when calculating $\nabla L$.

$$
\begin{gathered}
\nabla L=h_{d} \div \frac{A_{d}}{w_{d}} \\
h_{d}=\frac{1}{2 l} \times\left(\sum_{j=\text { left }_{d}-l}^{\text {left }_{d}} \sum_{i=1}^{m} \operatorname{MRFI}(i, j)+\sum_{j=\text { right }_{d}}^{\text {right } t_{d}+l} \sum_{i=1}^{m} \operatorname{MRFI}(i, j)\right)
\end{gathered}
$$

If $\nabla L$ is greater than the preset threshold, this block is identified as a dental calculus block and marked on the original image, as shown in Fig. 4(b).

Method (2): Texture Analysis-Entropy and Slope. The dentin block with enamel or $A R$ greater than the threshold [Fig. 4(c)] is substituted in the gray level of $N I$ and binarized. It is observed that the gray scale distribution of dental calculus is more dispersed than that of the enamel surface layer, as shown in Fig. 4(d). The general tooth tissues and dental calculus can be distinguished after the gray scale distribution is analyzed. The intensity threshold is fixed at 220. In order to describe the feature information quantitatively, the gray-level co-occurrence matrix of texture analysis is used to obtain the entropy. The gray-level co-occurrence matrix describes the spatial texture as the probability of different gray levels being adjacent to each 


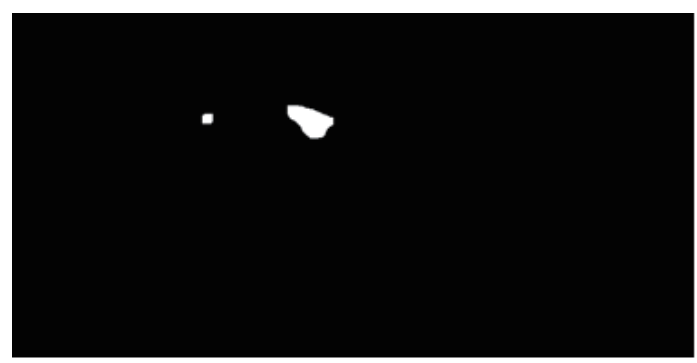

(a)

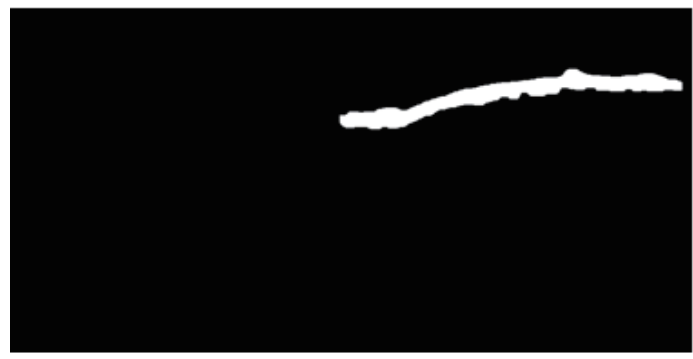

(c)

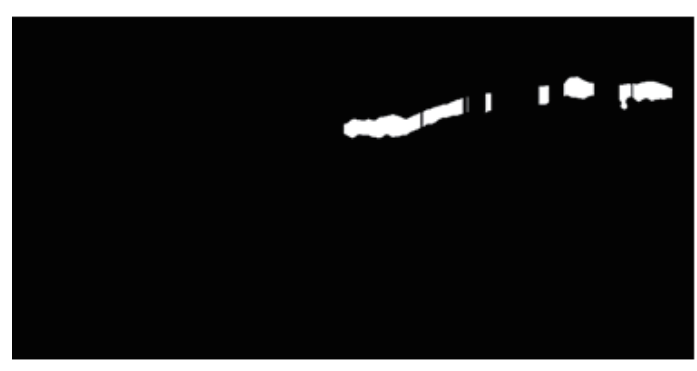

(e)

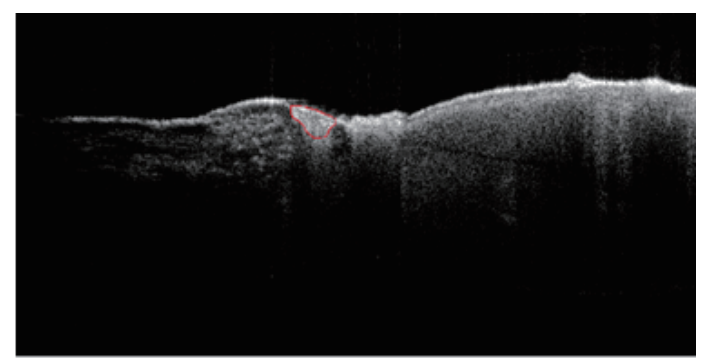

(b)

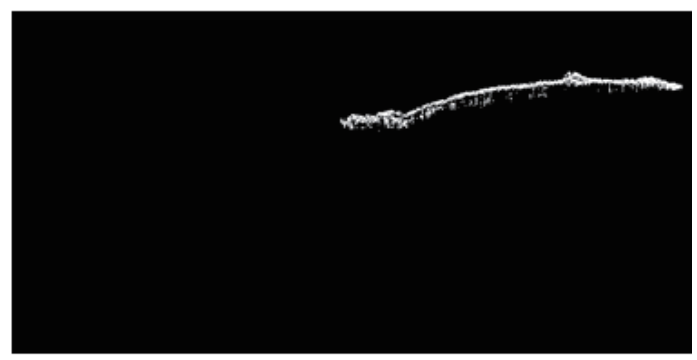

(d)

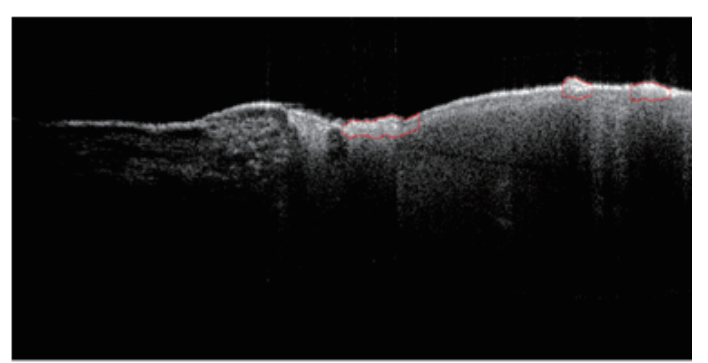

(f)

Fig. 4. (Color online) Results of each step in the process of segmenting dental calculus. (a) Block $A R<5$. (b) Result of dental calculus segmentation by method (1). (c) Block $A R>5$. (d) Binary image. (e) Entropy screening result $E>1.65$. (f) Result of dental calculus segmentation by method (2).

other, where $g i$ and $g j$ represent the gray levels of pixels, and the value on the matrix is the probability of two pixels in the image having the same gray level satisfying the preset direction and distance. The entropy $E$ is expressed as

$$
E=\sum_{g i=0}^{255} \sum_{g j=0}^{255} G(g i, g j) \times \log _{2}(G(g i, g j)) .
$$

If $E$ obtained from the mask centered on each pixel is greater than the preset threshold, the region of the same row is reserved, as shown in Fig. 4(e). Finally, if the tooth surface of the residual segment has a slope variation, it is identified as a dental calculus block and marked on the original image, as shown in Fig. 4(f). 


\section{Results and Discussion}

The OCT system used in this study has a light source with a center wavelength of 1060 $\mathrm{nm}$, a scanning area of $5 \times 15 \mathrm{~mm}^{2}$, a longitudinal resolution of $20 \mu \mathrm{m}$, a scanning frequency of $30 \mathrm{~Hz}$, a scanning depth of $3 \mathrm{~mm}$, and a spatial resolution of $968 \times 492$ pixels. To evaluate the performance of the segmentation algorithm, the assessment was performed using the mean manually delineated boundary of each area of dental calculus in the OCT image. Each area of dental calculus was demarcated by two graduate students and confirmed by two experienced radiologists. The two graduate students had more than one year of experience in the segmentation of dental calculus in an OCT image. Figure 5 shows the segmentation results of dental calculus in an OCT image. Dental calculus [Figs. 5(c) and 5(d)] is marked on the OCT image [Figs. 5(a) and 5(b)] of a tooth sample [Fig. 6(a)]. Figures 5(e) and 5(f) show one set of manually delineated dental calculus boundaries.

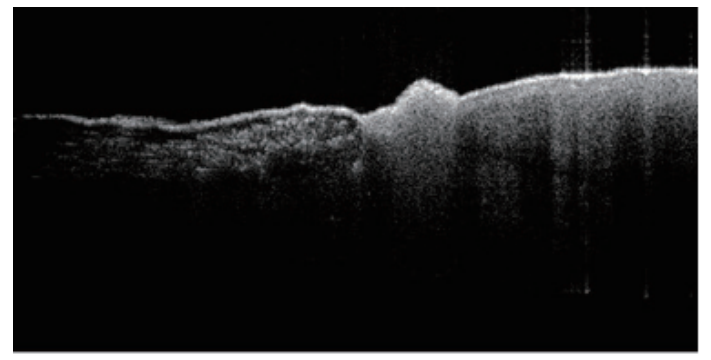

(a)

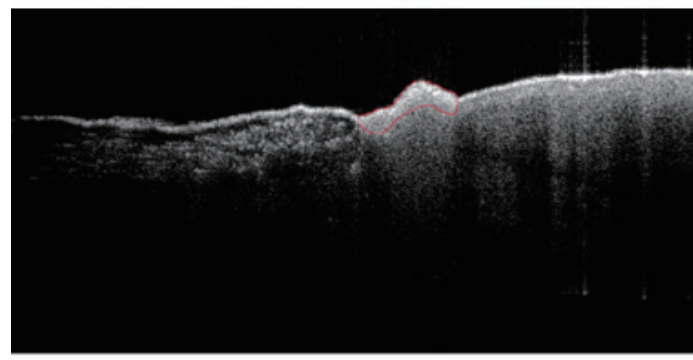

(c)

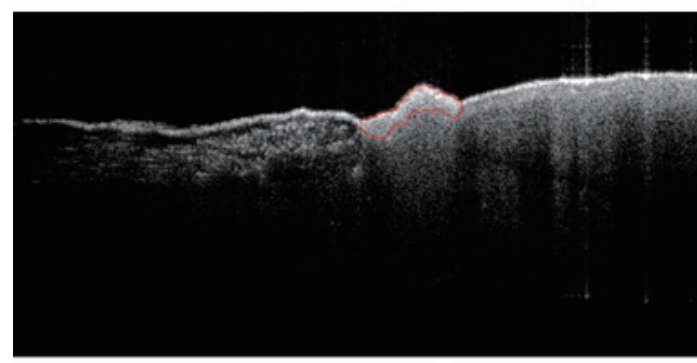

(e)

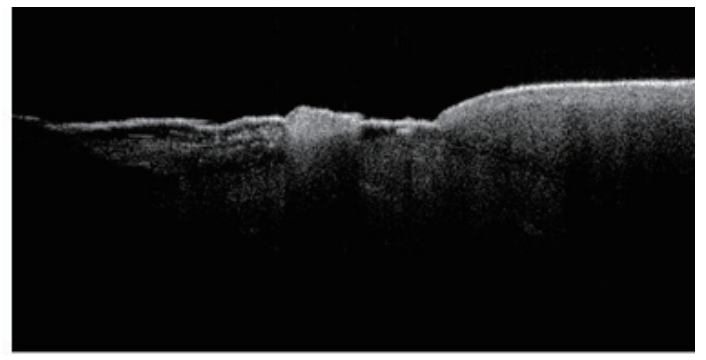

(b)

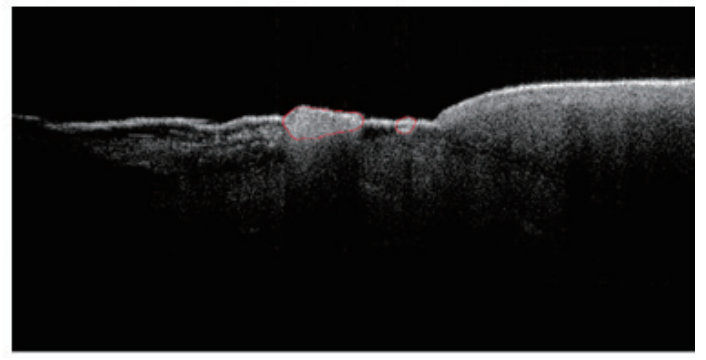

(d)

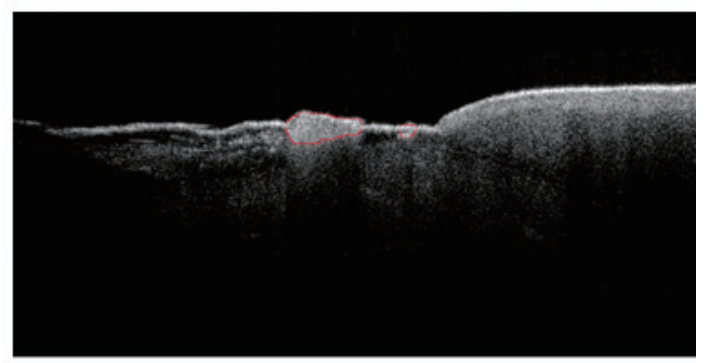

(f)

Fig. 5. (Color online) Results of dental calculus in OCT image. (a) and (b) Original image (two different cases). (c) and (d) Manually delineated boundaries for dental calculus. (e) and (f) Boundaries derived by the proposed algorithm. 


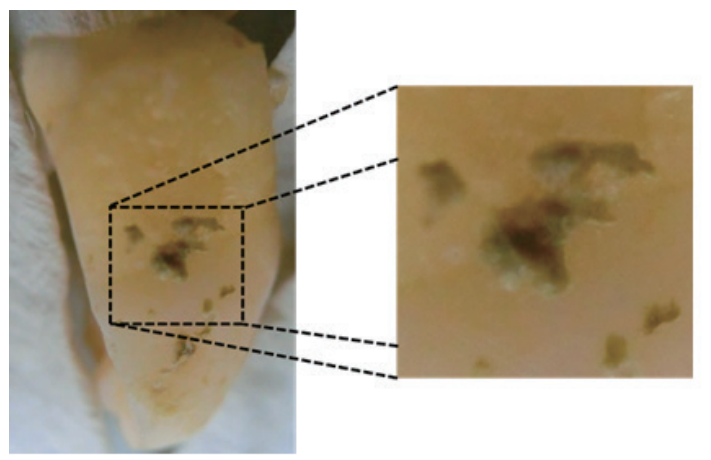

(a)

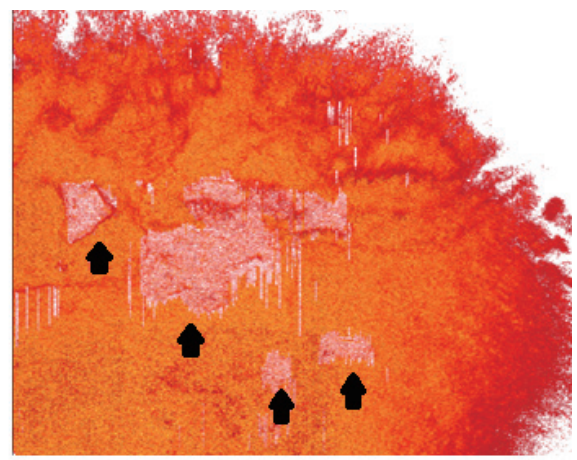

(b)

Fig. 6. (Color online) Segmentation result in the reconstructed 3D images. (a) Tooth sample. (b) Arrows show the segmentation result by the proposed algorithm in the reconstructed 3D images.

The mean distance between the boundaries generated by the proposed algorithm and the corresponding manually delineated boundaries was computed as $\nabla Z$, as expressed in Eq. (10). The column and row of the $g x$ th pixel on the boundary determined using the proposed algorithm are expressed as $A D_{i g x}$ and $A D_{j g x}$. The column and row of the gxth pixel on the manually delineated boundary are expressed as $M D_{i g x}$ and $M D_{j g x}$, where $g y=1,2,3, \ldots, g a$ and $g y=1,2,3$, ..., $g b$.

$$
\nabla Z=\frac{1}{g b} \times \sum_{g y=1}^{g b} \min _{g x} \sqrt{\left(A D_{i g x}-M D_{i g y}\right)^{2}+\left(A D_{j g x}-M D_{j g y}\right)^{2}}
$$

In 100 OCT images, there are 120 areas of dental calculus selected manually, 105 of which were identified correctly by the proposed algorithm. The sensitivity is $87.5 \%$, and the mean distance between the boundaries generated by the proposed algorithm and the corresponding manually delineated boundaries is $2.52 \pm 3.54$ pixels. Figure 6(a) shows the example of an actual case of dental calculus. To observe the segmented dental calculus more directly and easily, all 2D tooth images are reconstructed into 3D images. The arrows show the segmentation result in the reconstructed 3D images, i.e., the dental calculus region, in Fig. 6(b).

\section{Conclusions}

We proposed an automated dental calculus segmentation algorithm using OCT images. The proposed VITF solved the problem of gray scale differences on the tooth resulting from optical attenuation. The features of general tooth tissues and dental calculus were used to define the detection range using the K-means algorithm and MRF, and the candidate block of dental calculus was segmented by MM. The texture analysis showed that the features were successfully quantified, and the dental calculus region was recognized and segmented. The overall tooth structure and dental calculus information were displayed as a 3D reconstruction of 
the segmentation result. The evaluation sensitivity was $87.5 \%$ and the mean distance between the boundaries generated by the proposed algorithm and the corresponding manually delineated boundaries was $2.52 \pm 3.54$ pixels, proving the accuracy and reliability of the proposed segmentation algorithm. In this study, not only was a nonradiative and noninvasive detection mode obtained, but the symptom features were also quantified. Doctors no longer need to rely solely on their experience to recognize dental calculus, but can refer to specific data to assist in diagnosis.

\section{Declarations}

Author Contributions: Conceptualization, Chia-Yen Lee, Shyh-Yuan Lee, and ChingCheng Chuang; Funding acquisition, Chia-Yen Lee; Methodology, Guan-Jie Chen; Project administration, Chia-Yen Lee and Ching-Cheng Chuang; Resources, Chih-Chia Huang, and Shyh-Yuan Lee; Software, Guan-Jie Chen; Supervision, Chia-Yen Lee, Chih-Chia Huang, and Shyh-Yuan Lee; Validation, Guan-Jie Chen, Chih-Chia Huang, and Shyh-Yuan Lee; Writing original draft, Chia-Yen Lee; Writing - review \& editing, Yu-Hsien Lin.

\section{Conflicts of Interest}

The authors declare no conflict of interest.

\section{Acknowledgments}

This study was supported by the Ministry of Science and Technology, R.O.C. grant number: MOST 105-2815-C-239-009-E.

\section{References}

1 Ministry of Health and Welfare. 2007-2008 Investigation of Taiwan's population periodontal status and health behavior of over 18 years: http://www.mohw.gov.tw/cht/DOMHAOH/DM1_P.aspx?f_list_no=189\&fod_list_ no $=0 \&$ doc_no $=48300$

2 K. Pattanaporn and J. M. Navia: Periodontology 69 (1998) 955.

3 P. A. Testoni and B. Mangiavillano: World J. Gastroenterol. 14 (2008) 6444.

4 J. A. Evans, B. E. Bouma, J. Bressner, M. Shishkov, G. Y. Lauwers, M. M. Kenudson, N. S. Nishioka, and G. J. Tearney: Gastrointestinal Endoscopy 65 (2007) 50.

5 D. C. Fernández, H. M. Salinas, and C. A. Puliafito: Opt. Express 13 (2005) 10200.

6 R. J. Zawadzki, S. M. Jones, S. S. Olivier, M. Zhao, B. A. Bower, J. A. Izatt, S. Choi, S. Laut, and J. S. Werner: Opt. Express 13 (2005) 8532.

7 W. Drexler and J. G. Fujimoto: Opt. Express 27910 (2008) 45.

8 V. R. Korde, G. T. Bonnema, W. Xu, C. Krishnamurthy, J. R. Moore, K. Saboda, L. D. Slayton, S. J. Salasche, J. A. Warneke, D. S. Alberts, and J. K. Barton: Lasers Surg. Med. 39 (2007) 687.

9 T. Gambichlera, G. Moussaa, M. Sanda, D. Sandb, P. Altmeyera, and K. Hoffmanna: J. Dermatological Sci. 40 (2005) 85.

10 Y. S. Hsieh, Y. C. Ho, S. Y. Lee, C. C. Chuang, J. C. Tsai, K. F. Lin, and C. W. Sun: Sensors 13 (2013) 8928.

11 Y. S. Hsieh, Y. C. Ho, S. Y. Lee, C. W. Lu, C. P. Jiang, C. C. Chuang, C. Y. Wang, and C. W. Sun: J. Biomed. Opt. 16 (2011) 071409.

12 R. Maini and H. Aggarwal: Int. J. Image Process. 3 (2009) 1. 
13 K. He, J. Sun, and X. Tang: IEEE Trans. Pattern Anal. Mach. Intell. 35 (2013) 1397.

14 M. Abdullah-Al-Wadud, M. Kabir, M. Dewan, and O. Chae: IEEE Trans. Consum. Electron. 53 (2007) 593.

15 H. Ibrahim and N. S. P. Kong: IEEE Trans. Consum. Electron. 55 (2009) 891.

16 Z. Hou: Int. J. Biomed. Imaging ID 49515 (2006).

17 O. Nina, B. Morse, and W. Barrett: IEEE Workshop Applications of Computer Vision (Kona, HI, 5-7 January 2011).

18 L. Li, Y. Fu, P. Bai, and W. Mao: 3rd Int. Conf. Bioinformatics and Biomedical Engineering (Beijing, 11-13 June 2009).

19 D. Goshal and P. P. Acharjya: Int. J. Emerging Technol. Adv. Eng. 2 (2012) 373.

20 C. Li, C. Xu, C. Gui, and M. D. Fox: IEEE Trans. Image Process. 19 (2010) 3243.

21 F. Y. Shih and S. Cheng: Image Vision Comput. 23 (2005) 877.

22 N. Jifeng, W. Chengke, L. Shigang, and Y. Shuqin: Pattern Recognit. Lett. 28 (2007) 58.

23 A. K. Jain: Pattern Recognit. Lett. 31 (2010) 651.

24 B. C. Patel and G. R. Sinha: Int. J. Comput. Appl. 10 (2010) 35.

25 K. Sruthi: Int. J. Sci. Res. Publ. 3 (2013) 327.

26 Z. Kato and T. C. Pong: Image Vision Comput. 24 (2006) 1103.

27 A. Sopharaka, B. Uyyanonvaraa, S. Barmanb, and T. H. Williamsonc: Comput. Med. Imaging Graphics 32 (2008) 720 .

28 T Böhlke: Comput. Mater. Sci. 32 (2005) 276.

29 S. Lazebnik, C. Schmid, and J. Ponce: 10th IEEE Int. Conf. Computer Vision (17-21 October 2005$).$

30 M. Ushada, H. Murase, and H. Fukuda: Comput. Electron. Agric. 57 (2007) 149.

\section{About the Authors}

Chia-Yen Lee received her B.S degree in biomedical engineering from Chung Yuan Christian University, Chung Li, Taiwan, in 2004, and M.S. degree and Ph.D. degree in biomedical engineering from the National Taiwan University, Taipei, Taiwan, in 2005 and 2011, respectively. She is currently an assistant professor at the Department of Electrical Engineering, National United University, Miaoli, Taiwan. Her research interests include biomedical image processing, artificial intelligence in medical imaging diagnostics, medical imaging computeraided detection (CADe), computer-aided diagnosis (CADx), and medical device design and development.

Ching-Cheng Chuang received his B.S. and M.S. degrees from the Department of Biomedical Engineering, Chung Yuan Christian University, Taiwan, in 2003 and 2005, respectively. He received his Ph.D. degree from the Institute of Biomedical Engineering, National Taiwan University, Taiwan, in 2012. From August 2012 to January 2014, he was with the Biomedical Optical Imaging Lab, Department of Photonics, National Chiao Tung University, Taiwan, as a postdoctoral research fellow to study biomedical optical imaging techniques for clinical applications. In the summer of 2014, he joined the Computational Optics Group at University of Tsukuba, Japan, as a visiting scholar. Since 2014, he has been an assistant professor at the Institute of Biomedical Engineering, National Chiao Tung University, Taiwan. His current research interests include optical imaging techniques, functional near-infrared spectroscopy (fNIRS), optics in brain science, biomedical optical signal/image processing, biomedical optical device design, optogenetics, and clinical applications based on biomedical optical techniques.

Guan-Jie Chen received his B.S. and M.S. degrees from the National United University, Taiwan, in 2015 and 2017, respectively. His research interests are in digital image processing. 
Chih-Chia Huang received his B.S. degree from CSMU, Taiwan, in 1997 and his M.S. and Ph.D. degrees from the NTUMED, Taiwan, in 2007 and 2012, respectively. From 2013 to 2017, he was an assistant professor at Cardinal Tien Junior College of Healthcare and Management University, Taiwan. Since 2013, he has been the chief of the Dental Department, Cardinal Tien Hospital in the An Kang area of Xindian district. His research interests are in oral radiology and VRF teeth.

Shyh-yuan Lee received his DDS degree from National Yang-Ming University, Taiwan, in 1982, and his Prosthodontics Certificate and DScD degree from the School of Dentistry, Boston University, in 1994 and 1996, respectively. From 1996 to 2003, he was an associate professor, and since 2003 he has been a professor at Yang-Ming University. His research interests are in dental materials and clinical dentistry.

Yu-Hsien Lin received his B.S., M.S., and Ph.D. degrees in electronics engineering from National Chiao-Tung University, Hsinchu, Taiwan, R.O.C., in 2001, 2002, and 2006, respectively. His Ph.D. dissertation research focused on the engineering and physics of advanced memory devices (in particular, nanocrystal based). From 2006 to 2010, he was with the Taiwan Semiconductor Manufacturing Company (TSMC), Taiwan, as a principle engineer working on research and design for $\mathrm{N} 40 / \mathrm{N} 20$ process integration and device performance improvement. In 2011, he joined the Department of Electronic Engineering, National United University, Miaoli, Taiwan. R.O.C., and he is currently a full professor. His research interests include novel nonvolatile memory devices, high-k dielectric materials for CMOS devices, and poly-Si thin-film transistors. 